\title{
Inflammation and Intracellular Exposure of Dolutegravir, Darunavir, Tenofovir and Emtricitabine in People living with HIV
}

\author{
Micol Ferrara ${ }^{1}$, Elena Salvador ${ }^{1}$, Alice Trentalange ${ }^{2}$, Chiara Alcantarini ${ }^{3}$, Mattia Trunfio ${ }^{4}$, \\ Elvira Stefania Cannizzo ${ }^{5}$, Valeria Bono ${ }^{6}$, Silvia Nozza ${ }^{7}$, Amedeo De Nicolò ${ }^{1}$, Alice \\ Ianniello $^{2}$, Elisa De Vivo ${ }^{2}$, Antonio D'Avolio ${ }^{1}$, Giovanni Di Perri ${ }^{2}$, Stefano Bonora ${ }^{8}$, Giulia \\ Marchetti $^{5}$, and Andrea Calcagno ${ }^{4}$ \\ ${ }^{1}$ University of Turin \\ ${ }^{2}$ Department of Infectious Diseases, University of Torino \\ ${ }^{3}$ Università degli Studi di Torino \\ ${ }^{4}$ University of Torino \\ ${ }^{5}$ University of Milan \\ ${ }^{6}$ Affiliation not available \\ ${ }^{7}$ San Raffaele Hospital \\ ${ }^{8}$ Department of Infectious Disases, University of Torino
}

January 3, 2022

\begin{abstract}
Background: Antiretroviral therapy reduces systemic inflammation and immune activation, but not to levels like HIV-negative. Limited drug penetration within tissues has been argued as potential mechanism of persistent inflammation. Data on the role of inflammation on plasma/intracellular (IC) pharmacokinetics (PK) of ARV drugs through to downregulation/expression of cytochrome P450 3A/membrane transport proteins are limited. Aim of this study was to investigate the correlation between inflammation markers and plasma/IC PK of different ARVs regimen in HIV-positive patients. Methods: We included in the study ART-treated HIV+ pts switching to 3 different ARV regimens: 1) DTG-based dual-therapy plus boosted-PIs, 2) DTGbased triple-therapy without PIs, 3) DRV/c-based triple-therapy. Plasma and IC ARV drugs concentration means at the end of dosing interval (T0), IM on samples concomitantly with ARV PK determination: sCD14, CRP, IL-6 and LPS were analysed. Results: 60 samples from pts included in the switching study were used for measuring plasma and IC concentrations of HIV drugs. No significative differences between CRP, sCD14, IL-6 and LPS values in 3 arms of therapy were observed. Significant correlation was observed between tenofovir plasma concentrations and sCD14 $(\mathrm{p}<0.001)$, DRV plasma concentration and sCD14 $(\mathrm{p}=0,07)$ and DRV IC/plasma ratio and Log10 IL-6 concentrations $(\mathrm{p}=0.04)$. Furthermore, in 24 pts on DTG-TT, we observed a negative trend between DTG IC concentrations and $\mathrm{sCD} 14(\mathrm{p}=0.09)$. Conclusions: Our preliminary data support the hypothesis of lower IC concentrations of DRV and DTG in pts with higher plasma IM, suggesting an interplay between HIV drug penetration and persistent inflammation in cART-treated HIV-positive patients.
\end{abstract}

Inflammation and Intracellular Exposure of Dolutegravir, Darunavir, Tenofovir and Emtricitabine in People living with HIV

M. Ferrara ${ }^{1 *}$, E. Salvador ${ }^{1}$, A. Trentalange ${ }^{1}$, C. Alcantarini $^{1}$, M. Trunfio ${ }^{1}$, E. S. $_{\text {Cannizzo }}{ }^{2}$, V. Bono $^{2}$, S. Nozza $^{3}$, A. De Nicolò ${ }^{4}$, A. Ianniello ${ }^{4}$, E. De Vivo ${ }^{4}$, A. D'Avolio ${ }^{4}$, G. Di Perri ${ }^{1}$, S. Bonora $^{1}$, G. Marchetti ${ }^{2}$, A. Calcagno ${ }^{1}$ 1.Unit of Infectious Diseases, Department of Medical Sciences, University of Turin, 10149 Turin, Italy; 2. Department of Health Sciences, Clinic of Infectious Diseases, ASST, Santi Paolo e Carlo, University of Milan, 20142 Milan, Italy ; 3. Department of Infectious Diseases, 
San Raffaele Scientific Institute, 20127 Milan, Italy; 4. Laboratory of Clinical Pharmacology and Pharmacogenetics, Department of Medical Sciences, University of Turin, 10149 Turin, Italy. ${ }^{*}$ Corresponding author:micol.ferrara29@gmail.com. Department of Medical Sciences, University of Turin, Corso Svizzera 164, 10149 Turin, Italy WHAT IS ALREADY KNOWN ABOUT THIS SUBJECT A valid intracellular penetration of antiretroviral drugs is required to reach drugs target for virological control. Penetration within reservoirs and in the intracellular compartment is mediated by membrane protein drug transporters and during chronic and systemic inflammation defence mechanisms are enhanced such as higher expression of drug transport membrane protein responsible of the uptake/excretion of antiretroviral drug with a potential reduction of antiretroviral concentration within cells. In this study we wanted to better evaluate the role of markers of inflammation on intracellular antiretroviral concentration. WHAT THIS STUDY ADDS We wanted to explore the potential interplay between inflammation and antiretroviral pharmacokinetics. Our preliminary results show for the first time how the penetration of some of the antiretrovirals in study is significantly lower in presence of higher plasma inflammation biomarkers.Background: Antiretroviral therapy reduces systemic inflammation and immune activation, but not to levels like HIV-negative. Limited drug penetration within tissues has been argued as potential mechanism of persistent inflammation. Data on the role of inflammation on plasma/intracellular (IC) pharmacokinetics (PK) of ARV drugs through to downregulation/expression of cytochrome P450 3A/membrane transport proteins are limited. Aim of this study was to investigate the correlation between inflammation markers and plasma/IC PK of different ARVs regimen in HIV-positive patients.Methods: We included in the study ART-treated HIV+ pts switching to 3 different ARV regimens: 1) DTG-based dual-therapy plus boosted-PIs, 2) DTG-based triple-therapy without PIs, 3) DRV/c-based triple-therapy. Plasma and IC ARV drugs concentration means at the end of dosing interval (T0), IM on samples concomitantly with ARV PK determination: sCD14, CRP, IL-6 and LPS were analysed.Results: 60 samples from pts included in the switching study were used for measuring plasma and IC concentrations of HIV drugs. No significative differences between CRP, sCD14, IL-6 and LPS values in 3 arms of therapy were observed. Significant correlation was observed between tenofovir plasma concentrations and sCD14 $(\mathrm{p}<0.001)$, DRV plasma concentration and $\mathrm{sCD} 14(\mathrm{p}=0,07)$ and DRV IC/plasma ratio and $\log _{10}$ IL-6 concentrations $(\mathrm{p}=0.04)$. Furthermore, in $24 \mathrm{pts}$ on DTG-TT, we observed a negative trend between DTG IC concentrations and sCD14 ( $\mathrm{p}=0.09)$. Conclusions: Our preliminary data support the hypothesis of lower IC concentrations of DRV and DTG in pts with higher plasma IM, suggesting an interplay between HIV drug penetration and persistent inflammation in cART-treated HIV-positive patients.BackgroundDespite the antiretroviral therapy (ART)-associated increase in life expectancy, people living with HIV (PLWH) continue to have increased morbidity and mortality compared to the general population, often due to nonAIDS-related events (1-3). There is evidence that heightened systemic inflammation and persistent immune activation, including monocyte activation, play an important role in the pathogenesis of various diseases (4-5). Reducing viremia below the limits of detection, regardless of the timing of ART initiation, has been associated with decline in immune activation (6), even though to an extent still higher than what is observed in accurately matched controls. Aim of ART is to maintain a persistent plasma undetectability and a valid penetration within intracellular (IC) compartment. Antiretroviral (ARV) drug targets are mostly intracellular, and different IC concentrations have been associated with distinct antiviral effects. The uptake and excretion of endogenous and exogenous compounds is expressed in the intestine, liver and kidney, and contribute to the intestinal absorption, liver metabolism and renal secretion of ionic drugs mediated respectively by drug transporters, belonging to solute carriers (SLC) or ATP-binding cassette (ABC) transporters. (7) The former is mediated in part by SLC transporters, such as organic cation transporter (OCT) $(8,9)$ organic anion transporter (OAT) $(10,11)$ and organic anion transporting polypeptides (OATP1B1, OATP1B3, and OATP2B1) $(12,13)$ located at the basolateral membrane of cells, while the latter is mediated by ABC transporters, such as P-glycoprotein (P-gp) encoded by multidrug resistance (MDR) 1 gene, multidrug resistance associated protein (MRP) 2, and breast cancer resistance protein (BCRP), located at the apical membrane of the cells (14). These results indicate that the expression levels as well as single nucleotide polymorphisms (SNPs) of drug transporters are responsible for individual variation in pharmacokinetics (15). Efflux transporters, such as P-gp, often protect from treatment cellular and anatomical reservoirs causing a decrease in IC drug concentrations. During chronic inflammation, cellular protective mechanisms are usually enhan- 
ced, such as with a higher expression of P-gp or organic anion transporters, and they have been associated with lower ARVs concentrations within cells, despite virological suppression (16). Even expression changes of many hepatic drug transporter genes have been observed in some pathophysiological conditions such as inflammation-induced primary biliary cirrhosis (PBC), and obstructive cholestasis. (16). Limited drug penetration within tissues and presence of immune sanctuaries has been argued as potential mechanisms of persistent inflammation (17). Few data are available on the role of inflammation on plasma and intracellular (IC) pharmacokinetics (PK) of ARV drugs through to downregulation of cytochrome P450 3A (Cyp 3A4), protein expression $^{1}$ and suppression of expression of P-gp in several tissues. (18) Therefore, aim of this study was to investigate the correlation between inflammation markers (IM), microbial translocation markers and plasma and IC PK of different ARVs regimen in stable HIV-positive patients (pts).Material and Methods We included in the study PLWH on stable ARV therapy with plasma HIV RNA $<50$ copies/mL since at least six months enrolled in different switch studies; all protocols received Ethics Approval and participants signed a written informed consent before being enrolled. Three different ARV regimens were assessed: i) Dolutegravir based dual therapy (DTG-DT) plus boosted Protease Inhibitors [PI, Atazanavir (ATV) or Darunavir (DRV)], ii) DTG based triple therapy (DTG-TT; without PI), and iii) DRV/c based triple therapy (DRV/c-TT). Pharmacokinetic analyses on plasma and intra-PBMCs ARV drugs concentration at the end of dosing interval $\left(\mathrm{C}_{\text {trough }}\right)$ were performed at the Laboratory of Clinical Pharmacology and Pharmacogenetics, University of Turin at the "Amedeo di Savoia" Hospital using UHPLC-MS/MS validated methods. We measured C-reactive protein (CRP), Inteleukin-6 (IL-6), soluble CD14 (sCD14) and Lipopolysaccharide (LPS) on frozen serum samples collected on the same day of PK measurement. For inflammation biomarkers analysis, samples were acquired on a Labscan 200 analyzer (Luminex, Austin, Texas) using Bio-Plex manager software (Bio-Rad, Hercules, California). Baseline demographics, clinical and immunological characteristics were described using median values (interquartile ranges) for continuous variables and number (percentage) for categorical variables. Non-compartmental plasma and IC PK parameters were calculated and reported as $\mathrm{ng} / \mathrm{ml}$ and expressed as geometric means (with 95\% confidence intervals, 95-CI). Non parametric analysis were performed for bivariate correlations (Spearman's rank correlation); all statistical analyses were performed using SPSS Statistics version 23 (IBM). Tests were all two-sided, and a $p$-value <.05 was considered statistically significant.Results Sixty pts were enrolled, respectively divided in three groups: 9 on DTG-DT, 25 on DTG-TT and 26 on DRV/c-TT. Participants were mostly male $(80 \%)$, with median age and BMI of 53 years (48-59) and $23 \mathrm{Kg} / \mathrm{m}^{2}$ (20-24). Median time on ART exposure and time from HIV diagnosis were respectively 22,5 (16-29) and 17,5 (12-23) years. At the time of the analysis $78,3 \%$ of pts had undetectable viremia (HIV-RNA $<20 \mathrm{cp} / \mathrm{mL}$ ) and lymphocytes CD4+ 598 (417-754) cells/ $\mu \mathrm{L}, 29(20-38) \%$ and, CD4+/CD8+ ratio 0,8 (0,4-1,1). $83 \%$ of pts presented at least one comorbidity, in particular $33 \%$ hypertension, $46 \%$ dyslipidemia, $26 \%$ hipovitaminosis $\mathrm{D}, 6 \%$ diabetes mellitus type $2,10 \%$ chronic kidney impairment. Median DTG and DRV plasma, IC and ratio IC/plasma PK were respectively $1308,4(808,2-1808,6) \mathrm{ng} / \mathrm{ml}$, $230.0(123.0-336,8) \mathrm{ng} / \mathrm{mL}$, ratio $0,174(0,127-0,221)$ and $1885,2(1316,9-2453,4) \mathrm{ng} / \mathrm{ml}, 829,0(489,5-1168,4)$ $\mathrm{ng} / \mathrm{mL}$ and ratio $0,436(0,283-0,588)$ (Table 1 with baseline and PK characteristics according to study arm). Median IM markers as sCD14, IL-6, CRP and LPS in DTG-TT, DTG-DT and DRV/c-TT arms were respectively 1.33 (1.08-1.58), 1.49 (1.29-1.69) and 1.39 (1.25-1.52); $2.33(0.82-3.85), 2.67$ (2.15-3.20) and 2.70 (0.52-4.88); 3.08 (0.97-5.2), $3.12(1.03-5.20)$ and 2.78 (0.33-5.22); $180.6(115.3-245.9), 163.0$ (113.3-212.7) and 171,6 (127.0-216.3) (Supplementary Table 1 - stratified by study arm) No significative difference in biomarkers among study arms was observed. We observed a significant inverse correlation between tenofovir and $\mathrm{sCD} 14$ concentrations $(\mathrm{rho}=-0.79, \mathrm{p}<0.001)$, between DRV and $\mathrm{sCD} 14$ concentrations $(\mathrm{rho}=0,31, \mathrm{p}=0,07)$ and between DRV IC/plasma ratio and $\log _{10}$ transformed IL-6 concentrations (rho $=-0.36, p=0.04$ ). In the 34 participants receiving DTG the drug IC concentrations were associated with age $(\mathrm{rho}=0.4, \mathrm{p}=0.01)$. Furthermore, in 24 pts on DTG-TT, we observed a trend towards lower IC DTG concentrations with higher $\mathrm{sCD} 14$ levels ( $\mathrm{rho}=-0.34, \mathrm{p}=0.09)$. Table 2 summarizes bivariate correlations (with rho and $\mathrm{p}$ values) while Figure 1 includes scatter-dot plots of these 4 described correlations.DiscussionIn our study we observed a correlation between some inflammation biomarkers and plasma and IC concentrations of the ARVs we assessed. In particular, sCD14 resulted to have different correlation with different ARVs: inverse and strongly significant with TDF plasma concentration, an inverse trend with DTG IC concentration when dosed with 
backbone in DTG-TT and a linear trend with DRV plasma concentration. Also IL-6 resulted to have an inverse and significant correlation with DRV IC/plasma ratio. By showing a negative correlation between plasmatic inflammatory biomarkers and ARV concentration, our study would altogether suggest a possible negative effect of a pro-inflammatory milieu on intracellular penetration of ARVs. Few manuscripts has so far analyzed the interactions between inflammation biomarkers and ARVs PKs, mostly focusing on ARVs penetration within tissue and its impact on viral parameters such as residual HIV viremia and reservoir (19) Moreover, data on the influence of different ART regimens on inflammation and immune activation have been controversial so far (20. Various authors reported different and mixed results on changes in systemic and vascular inflammation, monocyte activation, T-cell senescence and inflammation biomarkers based on the exposure to different ART regimens, as the comparison between a INSTI- vs NNRTI or PI-based ART (21-24). One of the potential mechanisms of HIV persistence is ongoing viral replication despite ART which can result from insufficient penetration of a number of antiretroviral drugs into tissues and anatomic sanctuaries (25). Importantly, heightened immune activation and inflammation has been previously suggested as both a cause and a consequence of HIV reservoir persistence and residual viral replication, mainly in tissues $(26,27)$. In chronic inflammatory conditions cellular protective mechanism are usually raised, such as a higher expression $\mathrm{P}-\mathrm{gP}$ or organic anion transporters, and they have been associated with lower ARVs concentrations within cells. Moreover naïve and memory T cells have been shown to differently express P-gP: those with higher expression can eliminate intracellular Raltegravir faster than those with low expression (16). In microglial cells, a dose dependent decrease in Saquinavir accumulation following treatment with LPS-induced inflammation was observed and on the other hand an accumulation of the same drug was increased significantly by a potent P-glycoprotein inhibitor (PSC833), confirming that the presence of neuroinflammation within the brain parenchymal compartment can exacerbate the ability to extrude antiretroviral agents from glial cells (28). Another instance has been shown in the context of liver cirrhosis, where a significantly decreased expression of mRNA levels of some drug transporters including OCT1 and OATP1B1 has been observed in cirrhotic (F4) versus non-cirrhotic patients (F0-F3). The reason could be explained with the presence of elevated levels of reactive oxygen species (ROS) and decreased antioxidant levels during HCV infection. In particular, ROS production within hepatocytes leads to the release of proinflammatory cytokines, such as tumor necrosis factor (TNF)-alpha, in turn decreasing mRNA levels of some SLC transporters including OCT1, OATP1B1, and OATP1B3, suggesting that TNF-a is in part responsible for the downregulation of SLC transporters in HCV-related cirrhosis (16). Our preliminary data support the hypothesis of lower IC concentrations of the ARVs analysed in patients with higher plasma IM, suggesting an interplay between HIV drug penetration and persistent inflammation in cART- treated HIV positive pts. We want to acknowledge the limitations of this work: the small sample size of the included participants was limited to those who underwent a TDM and inflammatory biomarkers analysis at the time of regular follow-up visits, retrospectively collected. Neither data on duration of virological suppression nor burden of comorbidities were available for the analysis. In the context of HIV curative interventions it will be important to include inflammatory biomarkers concomitantly to ARVs penetration in order to assess the role of inflammation/microbial translocations in HIV persistence and include appropriate interventions in such studies.Data availability statementResearch data are not sharedFunding This work was supported by Italian Ministry of Health - "Ricerca Finalizzata 2013" Grant. The funding sponsors had no role in: the design of the study; the collection, analyses or interpretation of data; the writing of the manuscript; or the decision to publish the results. Transparency Declaration None to declare . Bibliography 1. Samji H, Cescon A, Hogg RS, Modur SP, Althoff KN, Buchacz K, et al. Closing the gap: increases in life expectancy among treated HIV-positive individuals in the United States and Canada. PLoS One. 2013;8(12):e81355. [PMC free article] [PubMed] [Google Scholar] 2. Lewden C, Bouteloup V, De Wit S, Sabin C, Mocroft A, Wasmuth JC, et al. All-cause mortality in treated HIV-infected adults with CD4 [?]500/mm3 compared with the general population: evidence from a large European observational cohort collaboration. Int J Epidemiol. 2012;41(2):433-45. [PubMed] [Google Scholar] 3. Life expectancy of individuals on combination antiretroviral therapy in high-income countries: a collaborative analysis of 14 cohort studies. Lancet. 2008;372(9635):293-9. [PMC free article] [PubMed] [Google Scholar] 4. Bahrami H, Budoff M, Haberlen SA, Rezaeian P, Ketlogetswe K, Tracy R, et al. Inflammatory markers associated with subclinical coronary artery disease: the multicenter AIDS cohort study. J Am 
Heart Assoc. 2016;5(6):e003371. Large cross-sectional study from the MACS evaluating associations between inflammatory markers and subclinical vascular disease showing that higher IL-6, sICAM-1, sTNF-RI and -RII levels are associated with coronary stenosis in HIV-infected men. [PMC free article][PubMed] [Google Scholar] 5. Maisa A, Hearps AC, Angelovich TA, Pereira CF, Zhou J, Shi MD, et al. Monocytes from HIVinfected individuals show impaired cholesterol efflux and increased foam cell formation after trans endothelial migration. AIDS. 2015;29(12):1445-57. 6. Crowell TA, Fletcher JL, Sereti I, Pinyakorn S, Dewar R, Krebs SJ, et al. Initiation of antiretroviral therapy before detection of colonic infiltration by HIV reduces viral reservoirs, inflammation and immune activation. J Int AIDS Soc. 2016;19(1):21163. 7. Tsuji, A. and Tamai, I.: Carrier-mediated intestinal transport of drugs. Pharm. Res., 13: 963-977 (1996). 8. Inui, K., Masuda, S. and Saito, H.: Cellular and molecular aspects of drug transport in the kidney. Kidney Int., 58: 944-958 (2000). 8. Faber, K. N., Muller, M. and Jansen, P. L.: Drug transport pro- teins in the liver. Adv. Drug Deliv. Rev., 55: 107-124 (2003) 9. Jonker, J. W. and Schinkel A. H.: Pharmacological and physio logical functions of the polyspecific organic cation transporters: OCT1, 2, and 3 (SLC22A1-3). J. Pharmacol. Exp. Ther., 308: 2-9 (2004) 10. Chandra, P. and Brouwer, K. L.: The complexities of hepatic drug transport: current knowledge and emerging concepts. Pharm. Res., 21: 719-735 (2004) 11. Anzai, N., Kanai, Y. and Endou, H.: Organic anion transporter family: current knowledge. J. Pharmacol. Sci., 100: 411-426 (2006). 12. Hagenbuch, B. and Meier, P. J.: Organic anion transporting polypeptides of the OATP/SLC21 family: phylogenetic classifica- tion as OATP/SLCO superfamily, new nomenclature and molecular/functional properties. Pflua gers. Arch., 447: 653-665 (2004) 13. Mikkaichi, T., Suzuki, T., Tanemoto, M., Ito, S. and Abe, T.: The organic anion transporter (OATP) family. Drug Metab. Phar- macokinet., 19: 171-179 (2004) 14. Leslie, E. M., Deeley, R. G. and Cole, S. P.: Multidrug resistance proteins: role of P-glycoprotein, MRP1, MRP2, and BCRP (ABCG2) in tissue defense. Toxicol. Appl. Pharmacol., 204: 216-237 (2005). 15. Ogosawara et al Hepatitis C Virus-related Cirrhosis is a Major Determinant of the Expression Levels of Hepatic Drug Transporters Drug Metab. Pharmacokinet. 25 (2): 190-199 (2010). 16. Minuesa G. et al P-glycoprotein (ABCB1) activity decreases raltegravir disposition in primary CD41P-gphigh cells and correlates with HIV-1 viral load, J Antimicrob Chemother 2016; 71: 2782-2792 17. Fletcher, C.V et al. Persistent HIV-1 replication is associated with lower antiretroviral drug concentrations in lymphatic tissues. Proc. Natl. Acad. Sci. USA 2014, 111, 2307-2312.] 18. Srinivas N, Rosen EP, Gilliland WM Jr, Kovarova M, RemlingMulder L, De La Cruz G, White N, Adamson L, Schauer AP, Sykes C, Luciw P, Garcia JV, Akkina R, Kashuba ADM. Antiretroviral concentrations and surrogate measures of efficacy in the brain tissue and CSF of preclinical species. Xenobiotica. 2019 Oct;49(10):1192-1201. doi: 10.1080/00498254.2018.1539278. Epub 2018 Dec 17. PMID: 30346892; PMCID: PMC6579712. 19. Darcis G. Differences in HIV Markers between Infected Individuals Treated with Different ART Regimens. Viruses 2020, 12, 489;doi:10.3390/v12050489. 20. Hileman, C.O. et al Inflammation, Immune Activation, and Antiretroviral Therapy in HIV. Curr. HIV AIDS Rep. 2017, 14, 93-100 21. Morón-López, S et al. Switching From a Protease Inhibitor-based Regimen to a Dolutegravir-based Regimen: A Randomized Clinical Trial to Determine the Effect on Peripheral Blood and Ileum Biopsies From Antiretroviral Therapy-suppressed Human Immunodeficiency Virus-infected Individuals. Clin. Infect. Dis. 2019, 69, 1320-1328 22. Hileman, C.O. et al. Differential Reduction in Monocyte Activation and Vascular Inflammation With Integrase Inhibitor-Based Initial Antiretroviral Therapy Among HIV-Infected Individuals. J. Infect. Dis. 2015, 212, 345-354 23. Kelesidis, Tet al. Changes in Inflammation and Immune Activation With Atazanavir-, Raltegravir-, Darunavir-Based Initial Antiviral Therapy: ACTG 5260s. Clin. Infect. Dis. 2015, 61, 651-660 24. Kelesidis, T. et al. Changes in Markers of T-Cell Senescence and Exhaustion With Atazanavir-, Raltegravir-, and Darunavir-Based Initial Antiviral Therapy: ACTG 5260s. J. Infect. Dis. 2016, 214, 748-752.]. 25. Estes, J.D et al. Defining total-body AIDS-virus burden with implications for curative strategies. Nat. Med. 2017, 23, 1271-1276.; 18., 26. Hatano et al. Cell-Based Measures of Viral Persistence Are AssociatedWith Immune Activation and Programmed Cell Death Protein 1 (PD1)-Expressing CD4+ T cells. J. Infect. Dis. 2013, 208, 50-56 27. Klatt, N.R. et al. Immune activation and HIV persistence: Implications for curative approaches to HIV infection. Immunol. Rev. 2013, 254, 326-342. ] 28. Dallas et al Microglial activation decreases retention of the protease inhibitor saquinavir: implications for HIV treatment Journal of Neuroinflammation 2013, 10:58).

\section{Hosted file}


table inflammation paper.docx available at https://authorea.com/users/453538/articles/ 551357-inflammation-and-intracellular-exposure-of-dolutegravir-darunavir-tenofovir-andemtricitabine-in-people-living-with-hiv

\section{Hosted file}

Figure Inflammation paper.docx available at https://authorea.com/users/453538/articles/ 551357-inflammation-and-intracellular-exposure-of-dolutegravir-darunavir-tenofovir-andemtricitabine-in-people-living-with-hiv 